\title{
Relación entre asertividad sexual y autoeficacia para prevenir el VIH/SIDA en jóvenes universitarios del área de la salud
}

Relationship between sexual assertiveness and self-efficacy for preventing HIV/AIDS in young university students in the area of health care

Relação entre assertividade sexual e auto-eficácia na prevenção de HIV / AIDS entre estudantes universitários da área da saúde

Doi: http://dx.doi.org/10.23913/ride.v7i14.264

\author{
Andrés Osorio Leyva \\ Universidad Autónoma de Guerrero \\ osoriorenovacion@gmail.com
}

Alicia Álvarez Aguirre

Campus Celaya Salvatierra, Universidad de Guanajuato alicia.alvarez@ugto.mx

Verónica Margarita Hernández Rodríguez

Facultad de Enfermería, Universidad Autónoma de Querétaro covetojo@yahoo.com

Mercedes Sánchez Perales

Facultad de Enfermería, Universidad Autónoma de Querétaro sanchezpe.msp@gmail.com

Luz del Rosario Muñoz Alonso

Facultad de Enfermería, Universidad Autónoma de Querétaro charomualo@gmail.com 


\section{Resumen}

El objetivo de este trabajo de investigación es determinar la relación entre asertividad sexual y autoeficacia para prevenir el VIH/SIDA en jóvenes universitarios de la Unidad Académica de Enfermería $\mathrm{N}^{\circ}$ 2, en la Universidad Autónoma de Guerrero. Para ello se utilizó el método de estudio transversal, descriptivo y correlacional. Participaron 400 jóvenes universitarios por muestreo aleatorio simple, quienes respondieron una cédula con datos personales, escolares y laborales, una escala de asertividad sexual y una de autoeficacia para prevenir el VIH/SIDA. Se contó con la autorización de la institución educativa y el consentimiento/asentimiento informado de los participantes. En el análisis de los datos se obtuvo estadística descriptiva e inferencial. En cuanto al perfil sociodemográfico de los participantes, $50 \%$ son mujeres y el resto hombres, de los cuales $84.8 \%$ está soltero, $33.8 \%$ cursa el tercer semestre, $31.0 \%$ trabaja y estudia al mismo tiempo, y $65.8 \%$ estudia la licenciatura. Con respecto al nivel de asertividad sexual, $39.5 \%$ alcanzó un nivel promedio alto y $39.0 \%$ un nivel promedio alto de autoeficacia para prevenir el VIH/SIDA. Asimismo, hubo una relación significativa entre estas variables $(r=.406 ; p<0,01)$, lo que comprueba que a mayor nivel de asertividad sexual hay mayor nivel de autoeficacia para prevenir el VIH/SIDA. En conclusión, los resultados permitieron determinar que a mayor nivel de asertividad sexual hay mayor nivel de autoeficacia para prevenir el VIH/SIDA, lo cual sirve de base para diseñar intervenciones específicas en esta población de estudio.

Palabras clave: asertividad, autoeficacia, adolescente, adulto joven, salud sexual.

\section{Abstract}

The objective of this research is to determine the relationship between sexual assertiveness and self-efficacy for preventing HIV/AIDS in students of the Academic Unit of Nursing $\mathrm{N}^{\circ} 2$, at the Autonomous University of Guerrero. Cross-sectional, descriptive and correlational study method was used. Was attended by 400 students by simple random sampling, who answered a card with data personal, school, and work, sexual assertiveness scale and one of self-efficacy for preventing HIV/AIDS. Was authorized by the educational institution and the participants informed consent/assent. In the analysis of the data is obtained statistics descriptive and inferential. Out of the profile demographic of participants, 50\% are women and the rest men, of which $84.8 \%$ are unmarried, $33.8 \%$ is the third semester, $31.0 \%$ working and studying at the same time, and 65.8\% study Bachelor's degree. With respect to the level 
of sexual assertiveness, 39.5\% achieved a high average level and 39.0\% a high average level of self-efficacy for preventing HIV/AIDS. Also, there was a significant relationship between these variables $(r=.406 ; p<0.01)$, which noted that to higher level of sexual assertiveness no higher level of self-efficacy for preventing HIV/AIDS. In conclusion, the results allowed to determine that to higher level of sexual assertiveness have higher level of self-efficacy for prevention of HIV/AIDS, which serves as a base for designing targeted interventions in this study population.

Key Words: assertiveness, self-efficacy, teenager, young adult, sexual health.

\section{Resumo}

O objetivo desta pesquisa é determinar a relação entre assertividade sexual e auto-eficácia na prevenção de HIV / AIDS entre os jovens universitários unidade de enfermagem Academic No. 2, na Universidade Autónoma de Guerrero. Para este efeito, o método da transversal, foi utilizado estudo descritivo e de correlação. 400 estudantes universitários participaram por amostragem aleatória simples, que respondeu com uma cedula de dados pessoais, escolares e de trabalho, uma escala de assertividade sexual e auto-eficácia na prevenção de HIV / AIDS. Ele teve o apoio da escola e de consentimento / assentimento informado participantes. $\mathrm{Na}$ análise dos dados foram obtidos estatística descritiva e inferencial. Quanto ao perfil sóciodemográfico dos participantes, $50 \%$ são mulheres e os homens de descanso, dos quais $84,8 \%$ são solteiros, 33,8\% está no terceiro semestre, 31,0\% trabalham e estudam ao mesmo tempo, e 65,8\% estudando um grau. Quanto ao nível de assertividade sexual, 39,5\% alcançaram um elevado nível médio e 39,0\% maior nível médio de auto-eficácia para prevenir o HIV / AIDS. Houve também uma relação significativa entre estas variáveis $(r=406$;. $\mathrm{P}<0,01)$, o que prova que um maior nível de assertividade sexual não é maior nível de auto-eficácia para prevenir o HIV / AIDS. Em conclusão, os resultados permitiram determinar que um maior nível de assertividade sexual não é maior nível de auto-eficácia na prevenção de HIV / AIDS, que serve como base para a concepção de intervenções específicas na população estudada. Palavras-chave: assertividade, auto-eficácia, adolescentes, adultos, saúde sexual.

Fecha Recepción: Julio 2016 Fecha Aceptación: Enero 2017 


\section{Introducción}

La conducta se define como la manifestación de una respuesta observable hacia una situación específica (Fishbein y Ajzen, 1991). Para fines de esta investigación se tomó este concepto y se adaptó como conducta sexual del joven universitario. La conducta sexual abarca las acciones que el joven decide realizar en situaciones sexuales donde los estímulos que recibe tienen un papel muy importante, además del grado de coherencia entre pensamiento, sentimiento y acciones, ya que influyen en la manera como decide conducirse tras analizar la información disponible (Fergos y Zimmerman, 2005).

La conducta sexual de riesgo en los adolescentes puede incrementar el índice de VIH/SIDA. Esta conducta está influida por diversos factores de riesgo, por lo que es imprescindible que el joven reciba educación sexual que le ayude a contrarrestar la influencia negativa del entorno y evitar las situaciones de riesgo.

Dos factores de protección en situaciones sexuales de riesgo son la asertividad sexual y la autoeficacia para prevenir el VIH/SIDA, el primero es definido como la capacidad para iniciar la actividad sexual, rechazar la actividad sexual no deseada, así como negociar las conductas sexuales deseadas, el empleo de métodos anticonceptivos y los comportamientos sexuales más saludables (Morokoff et al., 1997). El segundo se define como la percepción que el joven tiene de su competencia para desarrollar una conducta sexual eficaz y así prevenir el SIDA (López y Moral, 2001).

Existe literatura que señala como conductas sexuales de riesgo al sexo casual, la actividad sexual sin protección/bajo el efecto del alcohol y/o drogas, no usar condón, tener múltiples parejas sexuales, iniciar la vida sexual de manera temprana, entre otras (Forcada, et al., 2013; Espada, Morales y Orgilés, 2014). También mencionan la baja asertividad sexual y autoeficacia para la prevención del VIH/SIDA.

Diversos investigadores han estudiado la asertividad sexual y autoeficacia para prevenir el VIH/SIDA de manera individual. Por su parte, Ballester, Gil-Llario, Ruíz-Palomino y Giménez-García (2013), reportaron puntuaciones medias de autoeficacia en el uso del preservativo, mientras que Jenkins y Kennedy (2013) reportaron un nivel alto de asertividad en las dimensiones de inicio, rechazo, embarazo y enfermedades de transmisión sexual. Para 
Fontanilla, Bello y Palacio (2011) se presentó alta capacidad en la aserción sexual; asimismo, Uribe y Orcasita (2011) reportaron niveles promedio altos de autoeficacia para prevenir el VIH/SIDA.

El objetivo del presente estudio fue determinar la relación entre asertividad sexual y autoeficacia para prevenir el VIH/SIDA en jóvenes universitarios de la Unidad Académica de Enfermería $\mathrm{N}^{\circ}$ 2, en la Universidad Autónoma de Guerrero.

\section{PLANTEAMIENTO DEL PROBLEMA}

El Programa Conjunto de la Naciones Unidas sobre el VIH/SIDA, reportó en 2013 que hay 35 millones de personas con VIH y que 1.5 millones han muerto como consecuencia del SIDA. En el caso de Latinoamérica, 1.6 millones de personas tienen VIH y 47000 mueren por causas relacionadas con el SIDA (ONUSIDA, 2014).

El Sistema de Vigilancia Epidemiológica de VIH/SIDA reportó al término de 2014, 174564 casos de SIDA notificados y 9573 de casos nuevos diagnosticados de VIH y SIDA notificados. Los estados con la mayor tasa de casos nuevos diagnosticados de VIH son Yucatán, Quintana Roo, Campeche, Tabasco y Oaxaca; y con SIDA, Campeche, Guerrero, Quintana Roo, Chiapas y Yucatán. El sistema además reportó que la proporción de casos de VIH $(77.1 \%)$ y SIDA $(82.1 \%)$ en hombres es mayor que en las mujeres. Finalmente, las defunciones por SIDA en el 2013 fueron 4971 y la tasa de mortalidad por 100 mil habitantes en ese mismo año sumó 4.2 (ONUSIDA, 2014).

Específicamente en el grupo de jóvenes de 15 a 24 años de edad en el 2014 se reportaron 1 437 casos como seropositivos de VIH, de los cuales 1134 son hombres y 303 son mujeres; con respecto al SIDA se reportaron 734 casos: 619 hombres y 115 mujeres. Sobre la categoría de transmisión se reportaron 731 casos por vía sexual. De esto se puede mencionar que el grupo de personas más vulnerables para contraer esta enfermedad son los adultos jóvenes, debido que el tiempo entre adquirir el VIH y desarrollar el SIDA es de 5 a 10 años aproximadamente; se puede afirmar que la mayoría de estas infecciones ocurren durante la adolescencia (ONUSIDA, 2014). 
En el estado de Guerrero se tienen 8216 casos notificados de SIDA al 2014, de los cuales 6 223 son hombres y 1993 mujeres, encontrándose una proporción de casos con respecto al total de $4.7 \%$. Este dato lo ubica por debajo de la Ciudad de México (15.1\%), Estado de México (10.5\%), Veracruz (8.9\%), Jalisco (7.3\%) y Chiapas (5.0\%) (ONUSIDA, 2014).

Ante este contexto es importante prevenir la conducta sexual de riesgo en los adolescentes y jóvenes universitarios, así como identificar la asertividad sexual y autoeficacia para prevenir el VIH/SIDA y con ello favorecer un estilo de vida promotor de la salud.

\section{MATERIAL Y MÉTODOS}

Se realizó un estudio de corte transversal descriptivo correlacional para describir las variables de asertividad sexual y autoeficacia y así prevenir el VIH/SIDA. Asimismo, se estudiaron las relaciones entre las variables antes señaladas y se recabó la información en un periodo determinado.

El universo lo conformaron los estudiantes de la Unidad Académica de Enfermería $\mathrm{N}^{\circ} 2$ de la Universidad Autónoma de Guerrero. El tipo de muestreo fue de tipo probabilístico y el cálculo de tamaño de muestra se calculó con la fórmula para poblaciones finitas: $n=\left(Z^{2} \mathrm{pq}\right.$ $\mathrm{N}) /\left(\mathrm{Ne}^{2}+\mathrm{Z}^{2} \mathrm{pq}\right)$. Donde se consideró un nivel de confianza de $99 \%$, con un tamaño de muestra de 400 participantes. La selección de la muestra fue aleatoria simple y en ella se consideraron las listas proporcionadas por el departamento de servicios escolares.

Como criterio de selección se incluyó a los estudiantes de ambos sexos y de edad indistinta, aparentemente sanos, quienes firmaron un consentimiento informado. No fueron incluidos aquellos estudiantes que no se encontraban al momento de la toma de datos. El criterio de eliminación incluyó los instrumentos que no fueron contestados en su totalidad y los estudiantes que decidieron no continuar en el estudio.

Con el fin de obtener la información sociodemográfica se diseñó una cédula con preguntas sobre datos personales, escolares y laborales. En la medición de la variable asertividad sexual se utilizó la escala de asertividad sexual (SAS), creada a partir de la propia definición tanto semántica como sintáctica (Carretero-Dios y Pérez, 2005) y del constructo definido por Morokoff et al. (1997). La SAS está compuesta por 18 ítems que recogen los tres 
componentes que definen el constructo de asertividad sexual. La primera sub-escala (Inicio; ítems 1-6) evalúa la frecuencia en que una persona comienza una relación sexual y que acontece de forma deseada; la segunda (Rechazo; ítems 7-12) mide la frecuencia en que una persona es capaz de evitar tanto una relación sexual como una práctica sexual no deseada; la última dimensión (Embarazo y enfermedades de transmisión sexual; ítems 13-18) evalúa la frecuencia en que una persona insiste en usar métodos anticonceptivos de barrera de látex con su pareja. Todos los ítems se puntúan sobre una escala de respuesta tipo Likert entre 0 (Nunca) y 4 (Siempre). La mitad de los ítems están redactados de forma inversa $(3,4,6,8,10$, 11, 13, 14 y 16). Puntuaciones elevadas indican mayor asertividad sexual. El alfa de Cronbach reportado en un estudio en mujeres universitarias obtuvo valores de entre .71 y .85

Para la medición de la variable autoeficacia se utilizó la escala de Autoeficacia para prevenir el SIDA, tomando su formato del estudio de López y Moral (2001), conformada por 27 reactivos tipo Likert entre 0 (Nada Seguro) y 5 (Total Seguro), organizada en cuatro subescalas: la primera mide la capacidad para decir "no" a las relaciones sexuales en diferentes circunstancias (A1 a A11); la segunda evalúa la capacidad percibida para preguntar al compañero sobre las relaciones sexuales anteriores y otras conductas de riesgo, como el consumo de drogas (B1 a B4); la tercera cuestiona sobre la capacidad percibida para adquirir y utilizar correctamente el condón (C1 a C8), y la última aborda la capacidad para mantener la virginidad hasta el matrimonio, ser fiel a la pareja y platicar de sexo con los padres (D1 a D4). La interpretación de la puntuación total de la escala es de la siguiente forma: 1-48 Nivel muy bajo de autoeficacia, 49-71 Nivel bajo de autoeficacia, 72-94 Nivel promedio bajo de autoeficacia, 95-117 Nivel promedio alto de auto eficacia, 118-135 Nivel alto de autoeficacia. La escala se ha aplicado en población mexicana obteniendo un alfa de Cronbach de .89 .

Se solicitó autorización al Comité de Investigación de la Facultad de Enfermería de la UAQ y posteriormente a la institución donde se realizó el estudio. En la recolección de los datos participaron además del investigador dos asistentes previamente capacitados para este fin. Se solicitó la relación de los estudiantes en el área de subdirección académica de la Unidad Académica $\mathrm{N}^{\circ} 2$ de Enfermería de la UAGro., para realizar la selección de los participantes a fin de proporcionarles una invitación para una reunión de información del proyecto dentro de las instalaciones de la institución educativa. 
El día de la reunión se les explicó el proyecto y específicamente en qué consistía su participación, esperando una duración aproximada de 25 minutos.

Para el análisis de la información se generó una base de datos en el programa estadístico para las ciencias sociales - SPSS, versión 17. Se procedió a obtener las frecuencias, proporciones y porcentajes para las variables categóricas y para las variables numéricas se calcularon medidas de ubicación, tendencia central y variabilidad. Además se realizó la Prueba de Bondad de Ajuste de Kolmogorov-Smirnov con corrección de Lilliefors para contrastar la hipótesis de normalidad en la distribución de las variables continuas. Para las hipótesis se construyeron índices y se efectuaron contrastes de hipótesis con pruebas de correlación de Pearson.

La presente investigación se apegó a las disposiciones generales del Reglamento de la Ley General de Salud en materia de investigación para la salud en su reforma del 2014, que establece el respeto a la dignidad y protección de los derechos y bienestar del sujeto de estudio, proporcionar una explicación clara y completa respecto a la justificación y objetivos de la investigación (SSA, 2014).

\section{RESULTADOS}

En relación con las características de la población de estudio se presenta la tabla 1. 
Tabla 1. Caracterización de la población de estudio.

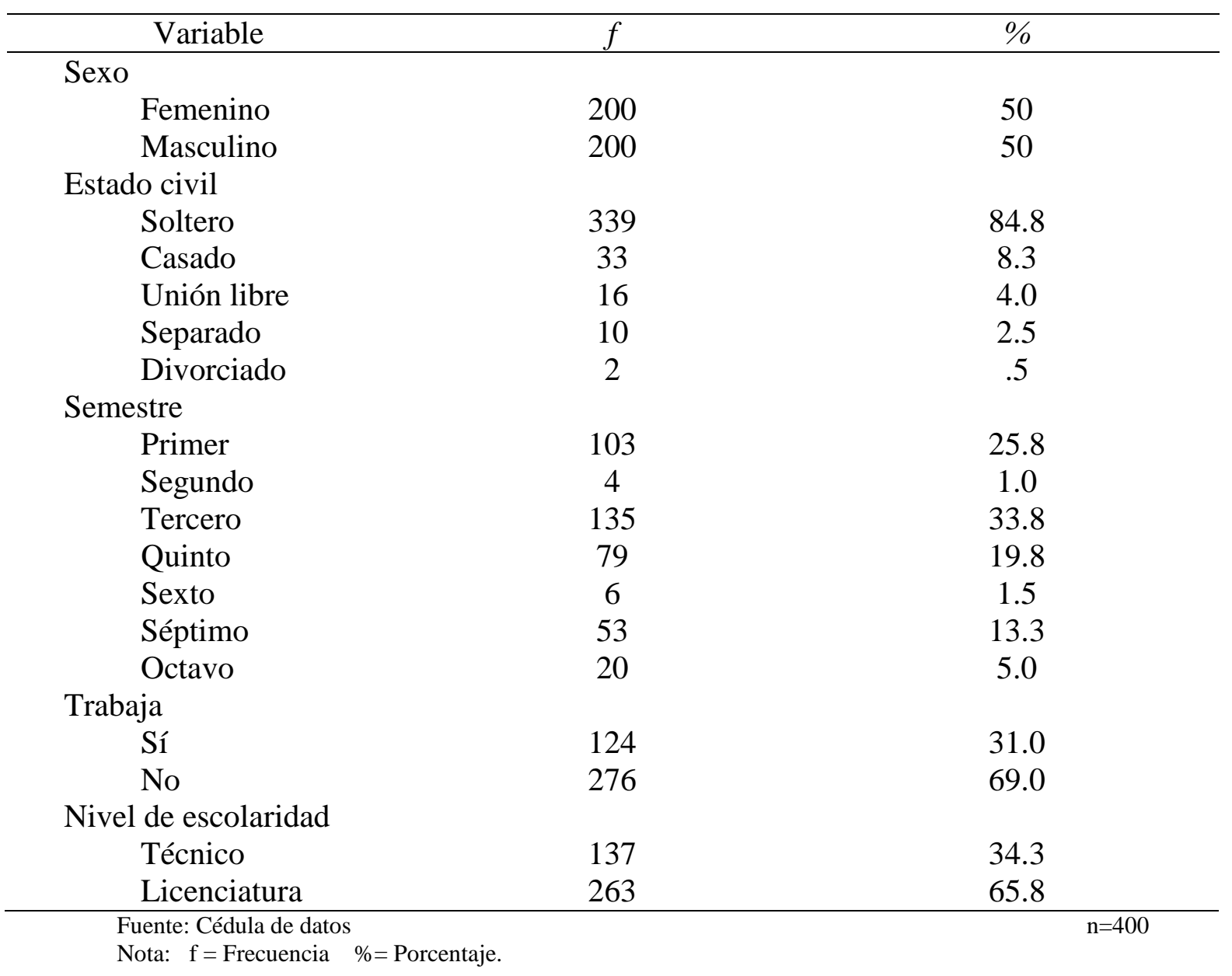

En la tabla 1 se muestran las características de la población de los 400 estudiantes participantes en el estudio, donde el género femenino es $50.0 \%$ del total de la población al igual que el masculino, de los cuales $84.8 \%$ es soltero, $33.8 \%$ cursa el tercer semestre, 31.0 $\%$ trabaja y estudia al mismo tiempo, y $65.8 \%$ estudia el nivel licenciatura.

Por otro lado, el nivel de asertividad sexual de los participantes se muestra en la tabla 2.

Tabla 2. Nivel de Asertividad Sexual

\begin{tabular}{lccc}
\hline \multicolumn{1}{c}{ Variable } & $f$ & $\%$ & \\
\hline Nivel muy bajo & 2 & .5 & \\
Nivel bajo & 25 & 6.3 & \\
Nivel promedio bajo & 153 & 38.3 & \\
Nivel promedio alto & 158 & 39.5 & \\
Nivel alto & 62 & 15.5 & \\
\hline Fuente: Escala de asertividad sexual & & & $\mathrm{n}=400$ \\
Nota: f = Frecuencia \%= Porcentaje. & & &
\end{tabular}


En la tabla 2 se muestra el nivel de asertividad sexual en frecuencia y porcentaje, ahí se observa que $39.5 \%$ de los participantes se encuentra en un nivel promedio alto y $15.5 \%$ en nivel alto con respecto a su asertividad sexual. Esto indica que son capaces de evitar tanto una relación sexual como una práctica sexual no deseadas y en su caso insistir en el uso de métodos anticonceptivos con su pareja, finalmente son capaces de tomar la decisión de comenzar una relación sexual si así lo desean.

La tabla 3 muestra el nivel de autoeficacia para prevenir el VIH/SIDA en los participantes.

Tabla 3. Nivel Autoeficacia para prevenir el VIH/SIDA en los participantes.

\begin{tabular}{|c|c|c|}
\hline Variable & $f$ & $\%$ \\
\hline Nivel muy bajo & 7 & 1.8 \\
\hline Nivel bajo & 48 & 12.0 \\
\hline Nivel promedio bajo & 104 & 26.0 \\
\hline Nivel promedio alto & 156 & 39.0 \\
\hline Nivel alto & 85 & 21.3 \\
\hline
\end{tabular}

Aquí $39.0 \%$ de los participantes presenta un nivel promedio alto de autoeficacia y $21.3 \%$ un nivel alto, lo que indica que poseen la capacidad para decir no a las relaciones sexuales en diferentes circunstancias y asimismo que son capaces de preguntar a su compañero/a sobre sus relaciones anteriores y otras conductas de riesgo como consumir drogas, adquirir y utilizar correctamente el condón, mantener la virginidad hasta el matrimonio, ser fiel a la pareja y platicar de sexo con los padres.

La tabla 4 muestra la relación que hay entre las variables asertividad sexual y autoeficacia para prevenir el VIH/SIDA en los participantes. 
Tabla 4. Relación de las variables asertividad y autoeficacia.

\begin{tabular}{llcc}
\hline \multirow{2}{*}{ Índice autoeficacia } & Correlación de Pearson & 1 & Asertividad \\
& Sig. bilateral & & .406 \\
& $n$ & 400 & .000 \\
Índice asertividad & Correlación de Pearson & .406 & 400 \\
& Sig. bilateral & .000 & 1 \\
& $n$ & 400 & 400 \\
\hline
\end{tabular}

** la correlación es significativa al nivel 0,01 (bilateral)

Aquí se observa que existe una relación significativa entre estas variables $(r=.406 ; p<0,01)$, lo que prueba que a mayor nivel de asertividad sexual hay mayor nivel de autoeficacia para prevenir el VIH/SIDA.

\section{DISCUSIÓN}

En el presente estudio fue factible la aplicación empírica de los conceptos teóricos de asertividad sexual y autoeficacia para la prevención del VIH/SIDA, conceptos base para el estudio de su relación en 400 estudiantes de la Unidad Académica de Enfermería No. 2 de la Universidad Autónoma de Guerrero.

El perfil demográfico de los participantes concuerda con el hecho de que están inscritos en la Unidad Académica de Enfermería N ${ }^{\circ} 2$ de la Universidad Autónoma de Guerrero. Además, muestra la igualdad de oportunidad de estudio tanto en hombres como en mujeres, lo que favorece la inclusión de género en la universidad. Por otro lado, 6 de cada 10 participantes sólo estudian se debe a que cuentan con el apoyo económico de su familia, mientras que 1 de cada 10 tiene una responsabilidad moral y económica con terceros por su condición civil.

Los resultados sobre asertividad sexual son similares a los reportados por Jenkins y Kennedy (2013), quienes encontraron un alto estado asertivo en el uso de condón para prevención de enfermedades y embarazos, así como alta asertividad sexual en la comunicación con los padres. Asimismo, los resultados encontrados por Fontanilla, Bello, Palacio (2011), reportan alta capacidad en la asertividad sexual, por lo que se puede afirmar que constituye un componente central de la sexualidad humana, ya que se relaciona con diversos aspectos de la respuesta sexual, como el deseo y la satisfacción sexual así como con menores niveles y frecuencia de victimización y coerción sexual y con la ausencia de conductas sexuales de 
riesgo. En este sentido, la asertividad sexual funciona como factor de protección ante las conductas sexuales de riesgo.

Los resultados de autoeficacia para prevenir el VIH/SIDA son similares a los reportados por Ballester, Gil-Llario, Ruíz-Palomino y Giménez-García (2013), quienes afirman que la mayoría de los participantes alcanzaron niveles promedio altos de autoeficacia. Lo mismo sucede con lo reportado por Uribe y Orcasita (2011) con respecto al nivel alto de autoeficacia frente al VIH/SIDA. Así, la autoeficacia para prevenir el VIH/SIDA tiene como elemento central las percepciones de los adolescentes sobre su capacidad de actuar, es decir, se sienten eficaces, son más resistentes a manifestar ciertas conductas, tienen metas más altas y persisten más en sus propósitos que aquellos que no tienen dicha capacidad. En este sentido, la autoeficacia funciona como factor de protección ante las conductas sexuales de riesgo frente al VIH/SIDA.

\section{CONCLUSIONES}

Los resultados obtenidos permitieron corroborar que a mayor nivel de asertividad sexual hay mayor nivel de autoeficacia para prevenir el VIH/SIDA. Adicionalmente, los instrumentos utilizados en el estudio Escala de asertividad sexual y Escala de autoeficacia para prevenir el SIDA mostraron ser bastante consistentes, por lo que se sugiere seguir utilizándolos en futuras investigaciones.

El instrumento de asertividad sexual se considera adecuado para la aplicación en jóvenes universitarios debido a la libertad de las preguntas sobre situaciones específicas relacionadas con su sexualidad. En México es inconsistente la facilidad que tiene la mujer joven para hablar sobre su sexualidad, por lo que se sugiere proteger la igualdad de género tomando la misma cantidad de hombres y mujeres.

Es necesario afrentar el problema con programas de prevención y acción para favorecer el nivel de asertividad sexual, con la finalidad de facilitar herramientas que promuevan la asertividad en el uso de condón y para comunicarse con sus padres, al mismo tiempo que ser autoeficaces para la prevención de VIH/SIDA. 


\section{Bibliografía}

Ballester, R., Gil-Llario, M., Ruíz-Palomino, E., y Giménez-García, G. (2013). Autoeficacia en la prevención sexual del SIDA: La influencia del género. Revista Anales de psicología, 29(1):76-82.

Carretero-Dios, H., y Pérez, C. (2005). Normas para el desarrollo y revisión de estudios instrumentales. Internacional Journal of Clinical and Health Psy-chology, 5:521551.

Espada, J., Morales, A., y Orgilés, M. (2014). Riesgo sexual en adolescentes según la edad de debut sexual. Acta Colombiana de Psicología, 17(1): 53-60. Recuperado de: http://www.scielo.org.co/pdf/acp/v17n1/v17n1a06.pdf

Fergus, S., y Zimmerman, M. (2005). Adolescent A framework for understanding healthy development in the face of risk. Annual Rewiew of Public Health, 26:399-419.

Fishbein, M., y Ajzen, I. (1991). The theory of planned behavior. Organizational Behavior and Human Decision Processes, 50:179-211.

Fontanilla, S., Bello A., J, Palacio. (2011). Conocimientos, habilidades de aserción sexual y toma de decisiones en función de la intención de los comportamientos sexuales y reproductivos en adolescentes. Psicogente, 14(26):294-309

Forcada, M., Pacheco, M., Pahua, M., Pérez, P., Todd, F., Pulido, Rull (2013). Conducta sexual de riesgo en estudiantes universitarios: factores de riesgo y protección Revista Intercontinental de Psicología y Educación, 15(1):23-46.

Jenkins, CC., y Kennedy, BR. (2013). An exploratory study of sexual assertiveness and characteristics of african american women in negotiating condon use at an HBCU. Journal of cultural Diversity, 3(20):139-145.

López, R.F., y Moral, R.J. (2001). Validación de una escala de autoeficacia para la prevención del SIDA en adolescentes. Salud Pública, 43:421-432.

Morokoff, P.J., Quina, K., Harlow, L.L., Whitmire, L., Grimley, D.M., Gibson, P.R., et al. (1997). Sexual Assertiveness Scale (SAS) for women: Developmrnt and validation. Journal of Personality and Social Psychology, 73:790-804.

ONUSIDA (2014). Resumen de la vigilancia epidemiológica del registro Nacional de Casos SIDA actualización al cierre 2014. 
Secretaría de Salud (2014). Reglamento de la ley General de Salud en Materia de Investigación para la Salud. Consultado el 20 de octubre de 2014. De: http://www.diputados.gob.mx/LeyesBiblio/regley/Reg_LGS_MIS_ref01_02abr14.do $\underline{\mathrm{c}}$

Uribe, S.F., y Orcasita, L.T. (2011). Evaluación de conocimientos, actitudes, susceptibilidades y autoeficacia frente al VIH/SIDA en profesionales de la salud. Avances en enfermería, 2 (XXIX), 271-284. 